\title{
Valoración del servicio al asegurado en la mejora de la gestión administrativa del Hospital General Milagro (IESS) en el año 2018.
}

\author{
Valuation of the service to the insured in the improvement of the \\ administrative management of the General Milagro Hospital (IESS) in the \\ year 2018.
}

Karina Salinas Muñiz. ${ }^{1}$, Huber Echeverría Vásquez. ${ }^{2}$ \& Miguel Cedillo Fajardo. ${ }^{3}$

DOI: https://doi.org/10.33262/visionariodigital.v3i2.410

\section{Resumen.}

El presente artículo de investigación toma el análisis de una institución pública que se encuentra en la ciudad de Milagro cantón de la provincia del Guayas y que se dedica a la actividad de prestar los servicios de salud pública como es el IESS, el enfoque esta dado en la valoración del servicio que los empleados prestan, para de esta forma tratar de mejorar la gestión administrativa al fin de buscar la satisfacción plena del cliente.

La investigación se basa en el servicio que presta la institución de esta forma poder medir el nivel de satisfacción de los clientes, el buen trato que lleve a elevar la imagen institucional buscando de una u otra forma ser el modelo que sirva y pueda ser implantado en las demás instituciones públicas, que tratan de buscar la forma de prestar el buen servicio, evitando largos tramites y esperas muchas veces innecesarias con lo que lograríamos establecer un modelo de gestión óptima para los clientes tanto internos como externos.

Palabras clave: Valoración, Gestión, Satisfacción del Cliente

\section{Abstract}

This research article takes the analysis of a public institution that is located in the city of Milagro canton of the province of Guayas and that is dedicated to the activity of providing public health services such as the IESS, the approach is given in the evaluation of the

\footnotetext{
1 Universidad Estatal de Milagro, Guayaquil, Ecuador, ksalinasm@unemi.edu.ec

2 Universidad Estatal de Milagro, Guayaquil, Ecuador, hecheverriav@unemi.edu.ec

3 Universidad Estatal de Milagro, Guayaquil, Ecuador, mcedillof@unemi.edu.ec
} 
service that the employees provide, in order to try to improve the administrative management in order to seek the full satisfaction of the client.

The research is based on the service provided by the institution in this way to measure the level of customer satisfaction, good treatment that leads to raise the institutional image looking in one way or another to be the model that serves and can be implemented in other public institutions, which try to find a way to provide good service, avoiding long procedures and often unnecessary waiting with what we would establish an optimal management model for both internal and external customers.

Keywords: Valuation, Management, Customer Satisfaction

\section{Introducción.}

El IESS es una institución de Salud que cuenta con 18.100 afiliados en los diferentes sectores de Milagro; esto representa el 0,7 afiliados a nivel nacional y el 2,4 afiliados de la provincia del Guayas en el año 2017, lo cual está bien distribuida en cada una de sus áreas y cuenta con un personal capacitado, sin embargo; la deficiencia comunicacional en atención al cliente que se maneja interna y externamente está afectando las relaciones entre usuarios, esta situación se origina debido a la inexistencia de una Jefatura de atención al público que permita optimizar la información tanto para el usuario interno como externo, generando una mala imagen a este Centro de Salud. (MIP, 2017)

Para los líderes del sector sanitario es un desafío mantenerse e instrumentar, de manera exitosa, sistemas que permitan una mejora en los servicios y al mismo tiempo, satisfacer las exigencias de los pacientes y demás usuarios ante los informes que se presentan sobre la calidad de la atención de salud. La calidad ante la atención medica representa el eje principal para los hospitales; tomando en cuenta que se convierte en uno de los puntos esenciales por parte de la Organización Mundial de la Salud (OMS, 2018), reúne distintas conceptualidades que se llevan generando con el transcurso del tiempo su aplicación ante la atención médica, sus dimensiones todo ello conforme a la opinión de los afiliados.

\section{Desarrollo.}

Según Orozco, Porro, \& Cubero (2010: 175) la valoración del servicio al asegurado se mide de acuerdo a tres dimensiones como las técnicas laborales y las relaciones en el trato al paciente asegurado. Esta acción es un elemento clave para la gestión y en especial para la mejora de sus procesos. No obstante, esta tarea no se realiza de un modo adecuado y llegar a un punto de invisibilidad dentro del mapa de prioridades institucionales (Murillo \& Saurina, 2013, pág. 304). Como se desarrolla en la presente investigación, lo cual permite analizar la valoración que existe ante la satisfacción del servicio en los asegurados del Hospital General de la Ciudad de Milagro. 
En Ecuador, los establecimientos de salud tanto en el sector público como en el privado enfrentan realidades parecidas y pese a los esfuerzos por brindar un mejor servicio, los usuarios siguen manifestando insatisfacción por la atención recibida; por parte del personal asistencial, técnico-administrativo, y la logística de los procesos. En este estudio se considera que el síntoma clave del problema es en la deficiente atención percibida por los afiliados, que hacen uso de los servicios que proporciona el hospital del IESS de la Ciudad de Milagro y desde nuestra perspectiva, se debe a que los empleados que se desempeñan en los diferentes cargos del hospital no han contado con una debida capacitación que les permita brindar una mejor atención al afiliado.

La mayoría de los afiliados están expuestos a prolongados tiempos de respuesta en el pedido de información y consultas médicas, que probablemente se debe a la extensa cobertura de la localidad y de sectores aledaños de la ciudad que atiende el Hospital IESS Milagro.

Esta investigación ayudara a conocer el diagnóstico de la calidad del servicio, realizar la contrastación empírica de la realidad con los paradigmas teóricos de calidad del servicio, satisfacción del usuario, así como indagar sobre su asociación. La originalidad y novedad de la investigación, se basa en que este estudio proporcionará nuevos conocimientos sobre la calidad de servicio y la satisfacción del usuario externo. (Mishima, Campos, Matumoto, \& Fortuna, 2016).

Evaluar la satisfacción de los usuarios, es relacionar mediante estrategias subjetivas de percepción analizando aquellos diferentes niveles que intervienen en la educación, de manera social y económica que influyen en el profesional ante los Centros de Salud de sus usuarios o afiliados. Entre los factores que permiten medir la calidad del servicio, se menciona: (Mishima, Campos, Matumoto, \& Fortuna, 2016)

El respeto y la dignidad a recibir un trato justo en todo momento y bajo toda circunstancia como un miembro del Hospital, establecer una identidad donde sea parte y el ente principal dentro de las consultas, permitiendo la seguridad de poder ser atendido dentro de las instalaciones del servicio, la comunicación entre los usuarios permitiendo ser completa y actualizada en las actividades de atención. Promoción y prevención y de esta manera el paciente pueda decidir su participación de manera voluntaria; es por ello que la ausencia de calidad origina un círculo vicioso que no es fácil librarse.

De acuerdo a estos puntos tomados en cuenta, la calidad de servicio de un Centro de Salud ante sus afiliados depende de las ambigüedades que se presentan en los servicios que brindan ante una atención medica más adecuada o indiferente; dependiendo de los valores en cuanto al estado y el contenido de la comunicación con el medio y el personal de salud, ante un servicio de baja calidad causando insatisfacción a los pacientes afiliados de los distintos sectores.

De acuerdo a Ortiz determina que cuando se detecta un bajo rendimiento dentro del Centro de Salud, significa que hay baja productividad en los programas que se desarrollan, afectando en los costos y reduciendo el presupuesto, generando falta de 
suministros, problemas en el mantenimiento de los equipos; afectando a los trabajadores del sector y su desmotivación y es allí donde afecta al cumplimientos de los objetivos que persigue los Centros de Salud porque ya no se trabajaría en equipo dando como resultado una mala calidad de la atención. (Ortíz, 2015)

Además se debe mejorar la capacidad del personal que labora, mediante un proceso de mejora continua y de calidad para las Instituciones, valiéndose de los resultados en los pacientes que consideran que la prestación de los servicios es de mala calidad y que no responde a sus necesidades; esto origina que los asegurados no concurran más al Hospital del IESS de Milagro.

La Base Legal de la institución señala como políticas el apego a las Normas, Leyes Institucionales, Reglamentos, y Resoluciones tanto de la Comisión Interventora así como las Resoluciones que emite el Consejo Directivo del IESS, para su aplicación y cumplimiento. Sus políticas se enmarcan dentro de: Administración de los programas de fomento y prevención de la salud., asistencia médica integral y de maternidad, atención odontológica preventiva y recuperativa, coordinar los programas gerontológicos, ejecutar el proceso de acreditación interna y externa, asistencia médica preventiva y curativa de hijos menores de 18 años, cónyuges o convivientes con derecho y administrar el presupuesto anual con análisis prospectivo y retrospectivo y subsidios monetarios transitorios y de maternidad.

Administración patrimonial y cumplir normas legales y procedimientos. También se ha realizado ejecuciones de planes de: Plan de Contingencia para casos de desastres, programa de desechos sólidos, etc. El sistema AS400 concesión de citas médicas a través del Call Center se implementó a partir del 20 de Mayo del 2009.

El Recurso Humano para fortalecer la cultura organizacional rica en valores, el abastecimiento de insumos de la determinación de cronogramas unificados de adquisición al 100\%. Equipamiento en el desarrollo de las unidades médicas para criterio de dotación, la Infraestructura de la gestión técnica de infraestructura a nivel provincial, descentralización, el financiamiento en el direccionamiento para la venta de servicios y desarrollar la autogestión sustentable y la Gestión de Aplicación y optimización del programa de gestión y desarrollo de unidades médicas (Resolución CD 082)

\section{La calidad en los hospitales}

Existen diferentes conceptos de lo que representa la calidad ya sea dentro de una organización como en el sistema educativo; comenzando por el mismo ser humano la calidad que lo identifica dentro de su entorno social, adaptándose al medio para satisfacer las necesidades de diferentes grupos de interés, pues para poder avanzar debe haber interés por parte de las empresas para que se refleje dentro la realidad organizacional.

La descripción del autor Guadalupe Fernández (2012) en cuanto a lo que representa la calidad menciona que "Es la adecuación constante y eficiente de nuestro producto ervicio a las expectativas que se les debe dar a los clientes convirtiéndose en una ventaja 
competitiva para los competidores" analizando los diferentes enfoques por los cuales la calidad toma importancia para las gestiones empresariales.

Tabla No. 1 Definiciones de diferentes autores sobre Calidad

Autores /año Concepto de la Calidad

Parasuraman,B. Zeit y L. Berry Es una acción que analiza lo esperado y lo percibido $(1985,1988)$ por las personas.

Berry (1988)

Kaoru Ishikawa (1988)

La calidad es un modo de servicio o previsión, un modo de pensamiento; mediante el desarrollo de nuevos servicios, nuevas políticas, nuevas tecnologías y nuevas instalaciones.

La calidad es el hecho de desarrollar, diseñar, manufacturar y mantener un producto que sea de calidad por el que identifica por su valor, su utilidad para el consumidor.

E.W. Deming (1988)

Define a la calidad como el grado de uso y a bajo costo de acuerdo a las necesidades del mercado para una mejora continua.

Fuente:https://www.nueva-iso-9001-2015.com/2016/09/desarrollo-concepto-calidad/

Estas ideas y definiciones de los diferentes autores se convierten en un centro de reflexión de lo que es la Calidad, el centro de atención en el interior de las organizaciones y que en varias ocasiones se convierten en aplicaciones inadecuadas dentro de la empresa. El interés de la calidad de la atención médica tiene su origen en el deseo de disminuir la desigualdad en el sistema de salud de los diferentes grupos sociales. Las instituciones de salud han planteado estrategias nacionales que pretenden el rescate de la calidad, lo que implicaría incremento en la satisfacción del usuario. (Villalba, 2013)

En el caso de las instituciones de salud su producto son servicios que tienen las características de ser intangibles y se pueden identificar dos tipos de calidad:

- Calidad técnica: es la aplicación de la ciencia y tecnología médica de forma que maximice los beneficios de la salud, sin aumentar de forma proporcional los riesgos en la atención mediante el cual se espera poder proporcionar al usuario 
externo el máximo y más completo bienestar, logrando un equilibrio más favorable de riesgo y beneficio.

- La calidad sentida: es la satisfacción razonable de las necesidades de los usuarios externos después de utilizar los servicios de calidad de la institución. Está en la subjetividad y debe ser explicada por ellos. Calidad es el cumplimiento de las normas técnicas y la satisfacción de las necesidades sentidas de los usuarios. La OPS/OMS propone como definición de calidad de las instituciones: (Ortega, y otros, 2012)

- Alto grado de satisfacción por parte de los usuarios externos.

- Un mínimo de riesgo para los usuarios externos e internos.

- Alto nivel de excelencia profesional.

- Uso eficiente de los recursos de las instituciones.

\section{La calidad del servicio}

Al tratar sobre calidad de servicio se toma en cuenta que su aplicación conlleva a varios conceptos establecidos por varios autores como: Fisher y Navarro (1994); Kotler (1997); Gronroos (1994) y Salvador (2005) que para ellos es un conjunto de actividades, beneficios o satisfactores que seran para su venta a travez del servicio que produce. Cuando uno adquiere un producto se analiza la calidad que aporta en lo fisico; en cambio en calidad del servicio se comprueba la manera como ese producto fue distribuido y que ventajas aporto ante su uso y si a cubierto todas las espectativas hacia el consumidor.

\section{Calidad de servicio hospitalario}

En primer lugar se toma en consideración lo que es la calidad, como un atributo del que cada persona posee en cuanto a sus intereses, costumbres y nivel educacional entre otros factores. En cuanto al servicio del paciente en un hospital se prestan las garantías necesarias para salvaguardar la integridad y salud de los pacientes otorgando una atención prioritaria permanente con un patrón alineado en la prestación médica de conocimiento y tecnología aplicando procedimientos adecuados en el diagnóstico y tratamiento.

Es por ello que para poder establecer la calidad medica recibida por el paciente se logra obteniendo evidencias que aboguen a la práctica médica y, servirá de manera útil y confiable para este tipo de investigación teniendo en cuenta siempre, el conocimiento del médico al paciente ante una relación de comunicación y aplicación para el beneficio del Hospital o Centro de Salud.

Finalmente, así como en la mayoría de los conceptos y realidades administrativas, cuando se dice de calidad en el servicio hospitalario pueden ser vistos, o bien como simples instrumentos, mecanismos de control, medios productores de certeza o criterios homogenizantes para lidiar con la complejidad, formas de gestionar la diversidad, criterios para dinamizar y adaptar las formas de movilizar la auto-organización de los servicios hospitalarios. (Jesus del Pilar \& Chico-Ruíz, 2014) 


\section{Satisfacción del usuario}

La definición es muy sencilla, el usuario está satisfecho si sus necesidades reales o percibidas, son cubiertas o excedidas, entonces ¿Cómo puede usted saber lo que el cliente necesita, quiere y espera? La satisfacción del usuario es un indicador importante de la calidad de servicios, no obstante resulta difícil de evaluarlo; los métodos y las medidas para la recolección de datos suelen influir en la respuesta del usuario, por lo tanto es recomendable hacerlo en forma privada y permanente. (Malagón-Londoño, Pontón, \& Reynales, 2016)

\section{METODOLOGÍA.}

Para el desarrollo del presente trabajo investigativo se ha empleado una metodología con profundidad descriptiva no experimental seccional, (Fontaines, 2012) afianzado por el método de análisis de datos textuales, que consiste en grosso modo en el conteo de las ocurrencias y apariciones de las unidades verbales en este caso utilizado en las encuestas realizadas mediante preguntas abiertas a los usuarios del Hospital General Milagro (IESS) en el servicio de consulta externa. Esta información se evidencia en la repetición constante de palabras, las mismas que van formando patrones relevantes evidenciando el valor del Chi cuadrado (X2) de cada palabra dentro de la clase agrupada.

\section{Ámbito de la investigación}

Siguiendo con la rutina investigativa, se desarrolló en el Servicio de Consulta Externa del Hospital General Milagro (IESS) ubicado en la ciudad de Milagro, Provincia del Guayas, Región 5, Ecuador. Esta unidad hospitalaria oferta servicios médicos para afiliados, jubilados, pensionistas y beneficiarios en Consulta Externa, Emergencia de adulto y pediátrica, Hospitalización y Servicios de Auxiliar y diagnóstico para el tratamiento, Unidad de Cuidados Intensivos y Quirófano, con capacidad resolutiva de 100 camas.

\section{Sujetos de Investigación}

Los sujetos tomados en cuenta para esta investigación fueron los usuarios que acuden diariamente para beneficiarse de los servicios que presta el hospital, agrupados en cuatro grupos, a saber: dependientes al Seguro Campesino, jubilados, pensionistas de Montepío y Voluntarios.

\section{Construcción del Corpus}

Para establecer el corpus que se va analizar, se utilizó el instrumento de la encuesta a través de un cuestionario o formulario compuesto de dos preguntas abiertas, las cuales estaban estructurada de la siguiente manera: a) Lo que más le disgusta del servicio recibido es....., b), Como mejoraría el servicio...., los usuarios fueron elegidos al azar, se obtuvieron 200 respuestas de 35 asegurados Campesinos, 46 pensionistas Jubilados, 8 asegurados Voluntarios, 11 pensionistas de Montepío, en un periodo de 5 días.

Los datos recabados fueron elaborados en una estructura textual para el respectivo análisis con la eliminación de signos gramaticales y reemplazo de letras evitando error de sintaxis por parte del software que se procede a detallar.

\section{Análisis del Corpus}

Una vez segmentado el contexto inicial de respuestas obtenidas de la encuesta realizada se procede al análisis utilizando un software específico para la estadística de datos 
textuales. Este programa se lo conoce como Iramuteq 0.7 alpha 2 para comprobar la teoría (Ratinaud) respecto a la lexicometría en donde las clases más representativas están en los valores altos del Chi-cuadrado $\left(\mathrm{x}^{2}\right)$, los resultados interpretados se observan a continuación.

\section{Resultados}

Del análisis estadístico textual mostrado por el software Iramuteq 0.7 alpha 2 (Ratinaud) se observa:

Número de textos 8

Número de ocurrencias: 2728

Número de formas: 278

Número de hápax: 17 (0.62\% de ocurrencias $-6.12 \%$ de formas)

Media de ocurrencia por texto: 341.00

En el gráfico 1 y tabla 1 se identifican seis clases agrupadas entre: uno y seis formando una sola unidad; cinco y cuatro de igual formando una sola unidad; estas dos unidades se entrelazan con las clases tres y dos, el enfoque de la valoración de los servicios analizados con respecto al mejoramiento se evidencia en las clases $1,4,5$ y 6 , en palabras cuyos chicuadrados son altos, donde la palabra afiliado $(\mathrm{X} 2=40.84 \mathrm{p}<0.00)$ y consulta $(\mathrm{x} 2=50.52$ $\mathrm{p}<0.00$ ) sustentan dicho enunciado

Gráfico 1 Factores de percepción de servicio al afiliado del Hospital de IESS de Milagro.

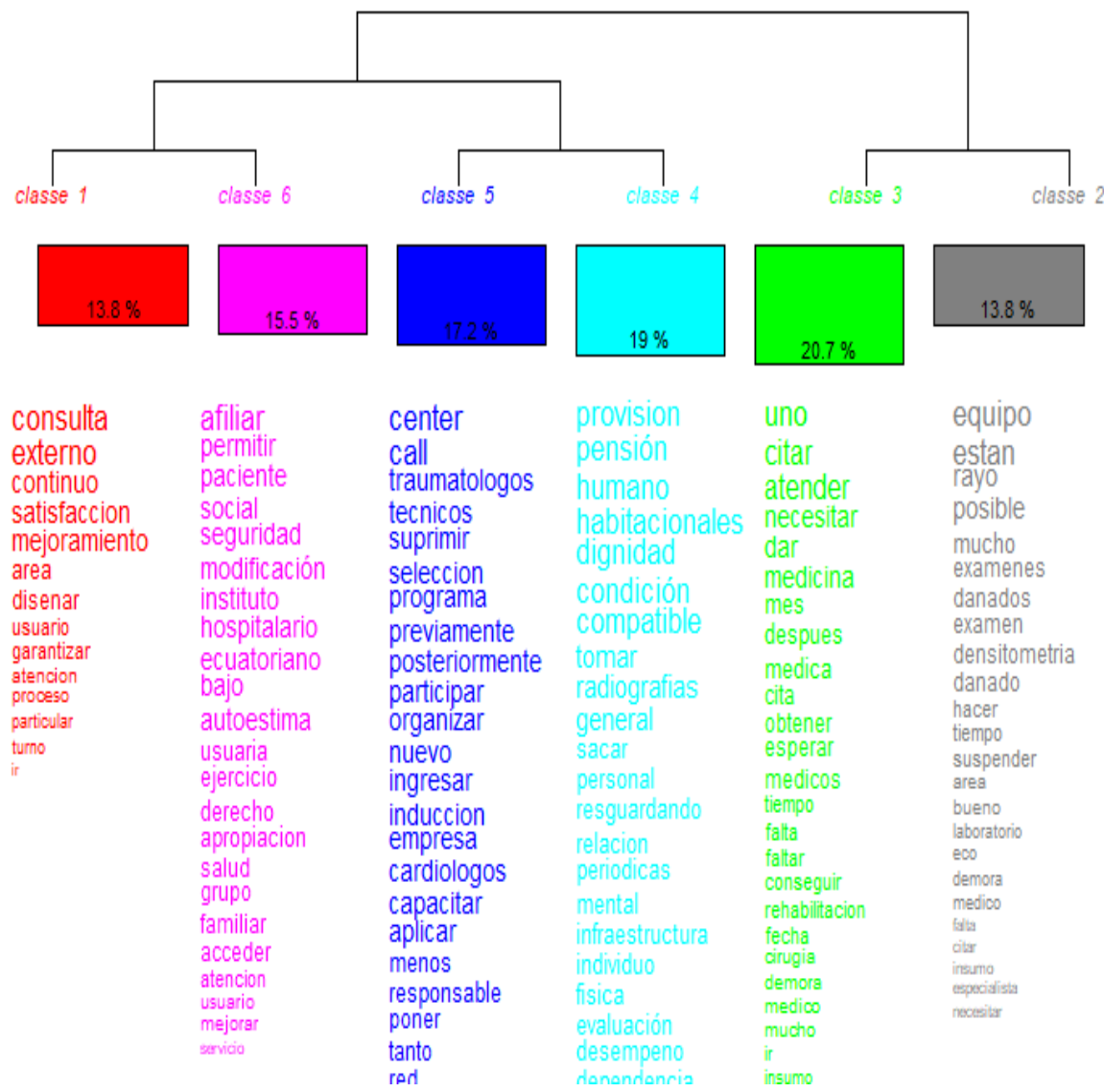

El enfoque de la valoración de los servicios analizados con respecto al mejoramiento se evidencia en las palabras cuyos chi-cuadrados son altos, donde la palabra afiliado $(\mathrm{X} 2=40.84 \mathrm{p}<0.00)$ y consulta $(\mathrm{x} 2=50.52 \mathrm{p}<0.00)$ sustentan dicho enunciado 
Tabla 1 Enfoque entre las clases y la valoración

\section{Clase 1}

\section{Palabra}

Tipo

Chi 2

$\mathbf{P}$

Consulta

Adjetivo

50.52

0.00

Satisfacción

Adjetivo

34.63

0.00

Clase 2

\section{Palabra}

Tipo

Chi 2

P

Equipo

Adjetivo

58.00

0.00

Dañado

Adjetivo

26.85

0.00

\section{Clase 3}

\section{Palabra}

Tipo

Chi 2

$\mathbf{P}$

Cita

Adjetivo

32.29

0.00

Medicina

Adjetivo

25.65

0.00

Clase 4

\begin{tabular}{llll}
\hline Palabra & Tipo & Chi 2 & P \\
Afiliado & Adjetivo & 40.84 & 0.00 \\
Derecho & Adjetivo & 23.39 & 0.00 \\
\hline
\end{tabular}

El enfoque de la valoración de los servicios analizados con respecto a la voz del usuario insatisfecho se evidencia en las clases 2 y 3 , en palabras cuyos chi-cuadrados son altos, donde la palabra equipo $(\mathrm{X} 2=58.0 \mathrm{p}<0.00)$; dañados $(\mathrm{x} 2=26.85 \mathrm{p}<0.00)$, citas $(\mathrm{x} 2=32.29$ $\mathrm{p}<0.00)$ y medicina $(\mathrm{x} 2=25.65 \mathrm{p}<0.00)$ sustentan dicho enunciado 
Gráfico 2 Diagrama semántica de factores de calidad de asistencia médica en el Hospital IESS (Milagro)

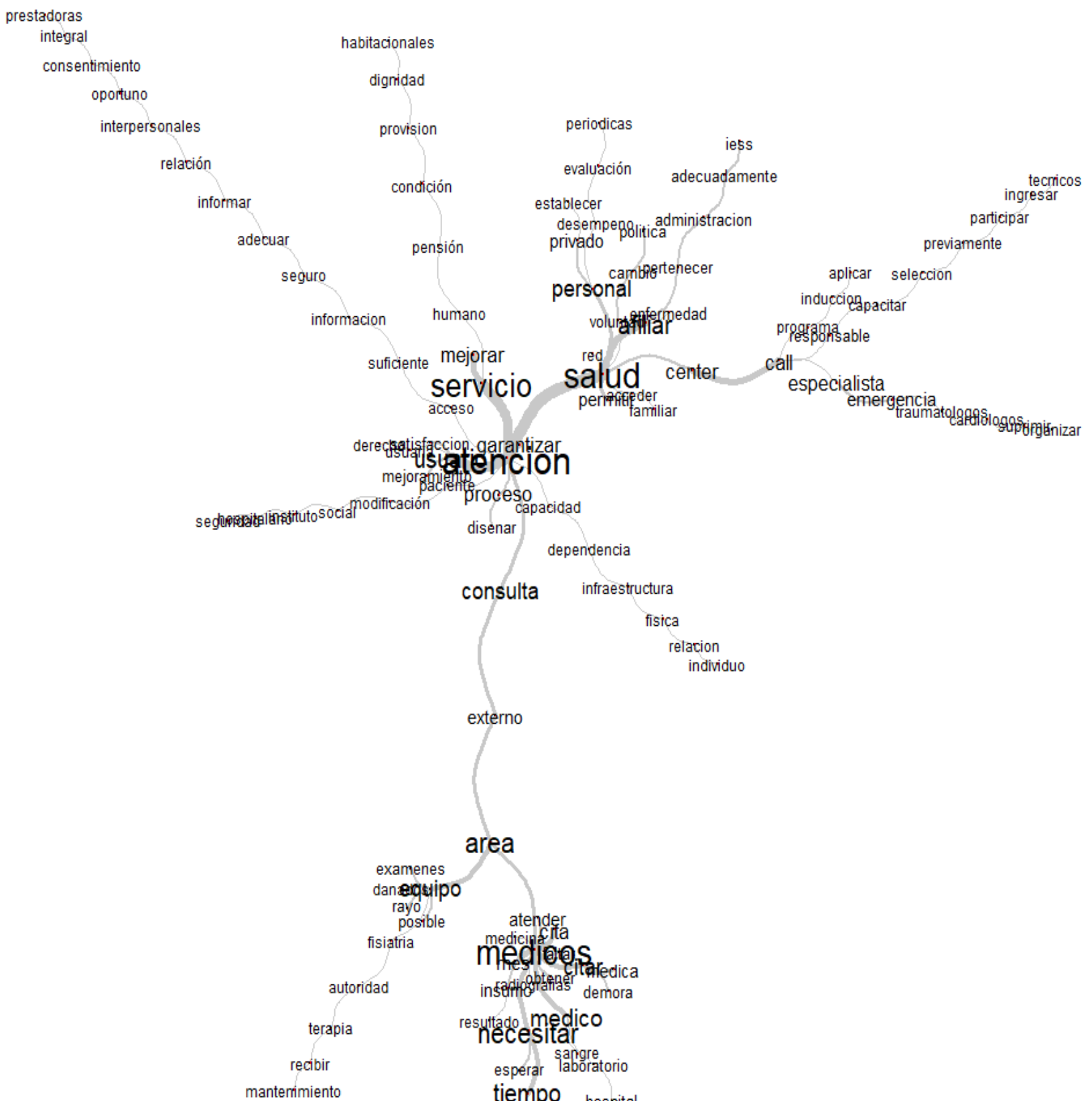

tam

seleccion

inducciopapacitar

programa porsable

especialista

Capacitar
emergencia
traumatoldeglos logorfirginizar

previamente 


\section{EI Hospital IESS (Milagro)}

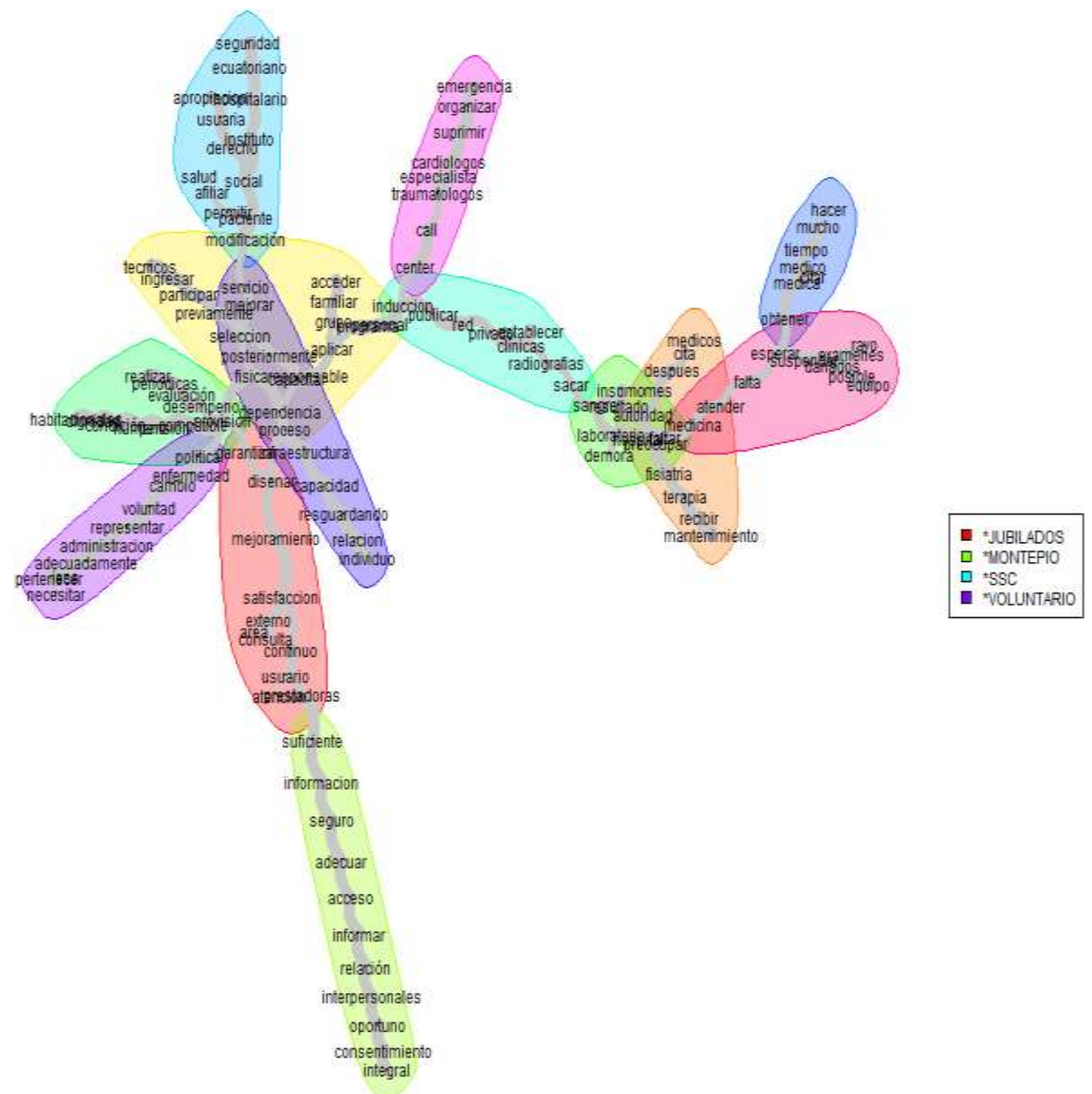

El método para poder medir la calidad ha sido a través de las respuestas que grosso modo en el conteo de las ocurrencias y apariciones de las unidades verbales en este caso utilizado en las encuestas realizadas mediante preguntas abiertas a los usuarios del Hospital General Milagro (IESS) en el servicio de consulta externa. Esta información se evidencia en la repetición constante de palabras, las mismas que van formando patrones relevantes evidenciando, lo cual permitirá demostrar que se haya actuado de una manera supuestamente de manera correcta, tratando de aprovechar las oportunidades que le ofrece 
el cambio innovador, contrarrestando las posibles amenazas que atacan al conjunto hospitalario ubicado en la Ciudad de Milagro.

Es por ello que esta investigación se llevó a cabo y es importante, porque permitió conocer el diagnóstico de la calidad del servicio, realizar la contrastación empírica de la realidad con los paradigmas teóricos de calidad del servicio, satisfacción del usuario, así como indagar sobre su asociación. La originalidad y novedad de la investigación, se basa en que este estudio nos proporcionará nuevos conocimientos sobre la calidad de servicio y la satisfacción del usuario externo. (Mishima, Campos, Matumoto, \& Fortuna, 2016)

De acuerdo a los resultados en las clases 1 y 4 a los factores antes indicados; se considera que existen muchos aspectos por los cuales las personas pueden dejar de frecuentar ciertos establecimientos de salud pública a pesar de ser gratuitos, uno de esos aspectos o factores sería el pésimo servicio que brinde un hospital. La migración de los pacientes es muy propensa a que suceda si los niveles de calidad de atención y servicios al cliente no se mejoran.

Además de tomar en cuenta que la población es probabilística de acuerdo a la cantidad de los diferentes grupos de afiliados, se procede a tomar una muestra simple aleatoria que mediante la técnica de la observación se toma a aquellas personas que estaban en ese momento en el pasillo de interconsulta y de atención medica; es por ello que fueron tomadas al azar de 200 personas consideradas para la recolección de los datos y describir los datos obtenidos en una estructura textual para el análisis estadístico mostrado por el software Iramuteq 0.7 alpha 2 (Ratinaud) ante la verificación de los resultados obtenidos.

\section{Conclusiones.}

- Posterior al análisis de los datos obtenidos y considerando que el objetivo del estudio es medir el valorar el servicio al asegurado de los pacientes que asistieron a la consulta externa en el Hospital General Milagro (IESS) unidad médica de segundo nivel de atención en salud, se puede concluir que la mayoría de los pacientes que participaron en esta investigación manifiestan su insatisfacción con relación a la percepción del servicio y evidenciada en respuestas las palabras de mayor pronunciamiento: las consultas médicas, equipos dañados, citas y medicina.

- De acuerdo a los resultados obtenidos se detectar algunos aspectos por los cuales las personas dejarían de frecuentar y afiliarse a ciertos establecimientos de salud debido al servicio que brinda el hospital, la migración de los pacientes a otros Centros de Hospitalización es muy propensa a que suceda si los niveles de calidad de atención y servicios al cliente no mejoran.

- Instaurar una reflexión midiendo la importancia que debe tener el usuario que se determinó ante la competencia médica, las condiciones físicas-técnicas, el grado de orientación e información y por último la atmosfera sociocultural del hospital, la cantidad de pacientes que eran atendidos en consulta externa, así como sus características, ante la necesidad de conocer la satisfacción mediante encuestas, 
cuyo valor psicométrico garantice la fiabilidad y validez, y adquirir gran parte de los conocimientos.

\section{Referencias Bibliográficas.}

Carmona-Calvo, M., Eva, M. S., Calvo-Mora, A., \& Priañez.Cristobal, R. (2015). Sistemas de gestión de calidad; un estudio en empresas del sur de España y norte de Marruecos. Elsevier, 9-3.

Fisher, L., \& Navarro, V. (1994). Instroducción a la investigación de mercado. México: McGraw-Hill Interamericana.

Fontaines, R. T. (2012). Formación de invstigadores educativos. Una visión racionalista. España: Editorial Académica Española.

Gronroos, C. (1994). Marketing y Gestión de servicios; la gestión de los momentos de la verdad y la competencia en los ervicios. Madrid. España: Editorial Diaz de Santos.

Guadalupe, F. (3 de Enero de 2012). Círculo de Economía de la provincia de Alicante. Obtenido de http://www.circuloeconomiaalicante.com/blog/un-concepto-decalidad-la-calidad-segunda-entrega-3-01-2012/

IESS. (2014). Hospital de IESS de Milagro. Babahoyo.

Jesus del Pilar, Y.-A., \& Chico-Ruíz, J. (2014). Nivel de satisfaccion del paciente asegurado en relación con la atención que brinda una clínica partitular en Trujillo, 2014. México: Sciendo.

Kotler, P. (1997). Mercadotécnia. México: Prentice-Hall.

Malagón-Londoño, G., Pontón, G., \& Reynales, J. (2016). Gerencia Hospitalaria para una adminsitración efectiva (Vol. 4). Bogóta-Colombia: Editoraial Medica Panamericana.

Mármol, M., \& Cruz, R. (2017). Factores relacionados con la satisfacción en consultas en hospitales de Madrid. REVENF(32), 12-1.

Merino Paza, M., Carrera Hueso, F., \& Roca Castelló, M. (2017). Relación entre la satisfacción laboral y la cultura de seguridad del paciente. Elsevier, 10.

Milagro, H. G. (2016). Manual de Calidad. . Milagro: MCA-SGC-001.

MIP. (2017). Ministerio de Industria y Productividad. Quito: Gobierno Ecuatoriano.

Mishima, S., Campos, A., Matumoto, S., \& Fortuna, C. (2016). Satisfacción del usuario bajo la perspectiva de la responsabilidad ?estratégia para análisis del sistemas universales? Revista Latinoaméricana de Enfermagem, 30-5. 
Murillo, C., \& Saurina, C. (23 de Octubre de 2013). Medida de la importancia de las dimensiones de la satisfacción en la provisión de servicios de salud. Elsevier, 4(27), 304-309. Obtenido de http://dx.doi.org/10.1016/j.gaceta.2012.12.015

Naranjo, E., \& Andrade, N. (2016). Proponer estrátegias para medir y mejorar la calidad en la atención de pacientes al servicio de fisioterapia en el Centro Médico Kinnemed. Guayaquil: Universidad de UDLA.

Ohlrost, F. (2012). Big Data Analytics. California: John Wiley\&Sons.

OMS. (19 de Octubre de 2018). "Pacientes en Defensa de su Seguridad" - Información general. Obtenido de http://www.who.int/patientsafety/patients_for_patient/statement/es/

Orozco, C., Porro, A., \& Cubero, O. (2010). Un sistema de gestión de calidad de salud, situación actual y perspectivas en la atención primaria. Revista cubana de Salud Pública(36), 175-179.

Ortega, C., Romero de Castilla, J., MAsilla, R., Quiroga, C., Gómez, B., \& Duran, T. (2012). Mejora de la calidad asistencial a los pacientes de la unidad de dolor tor+acico de un hospital comarcal. Elsevier Doyma, 4(25), 242-250.

Ortíz, G. (2015). Gerencia y Administración Estratégica de la Atención Médica. México: Panamericana.

Pública, M. d. (2014). Lineamientos operativos para la implementación del MAIS y RPIS. Quito: Dirección Nacional de NOrmatización.

Salud, L. O. (2012). Ley Orgánica de Salud. Quito: Rregistro Oficial.

Salvador, M. (2005). La percepción del cliente de los elementos determinantes de la calidad del servicio. Madrid: Papeles del Psicólogo.

Vallina, M. (2014). Marketing Digital. Madrid: Paranainfo.

Villalba, C. (2013). La calidad del servicio: un recorrido historico conceptual, sus modelos mas representativos y su aplicación en las universidades. Revista Dialnet, $3-20$.

\section{|| Ciencia}


Para citar el artículo indexado.

Salinas K, Echeverría H. \& Cedillo M. (2019). Valoración del servicio al asegurado en la mejora de la gestión administrativa del Hospital General Milagro (IESS) en el año 2018. Revista electrónica Visionario Digital 3(2), 213-227. Recuperado desde: http://visionariodigital.org

\section{Ciencia \\ LDigital}

El artículo que se publica es de exclusiva responsabilidad de los autores y no necesariamente reflejan el pensamiento de la Revista Ciencia Digital.

El articulo queda en propiedad de la revista y, por tanto, su publicación parcial y/o total en otro medio tiene que ser autorizado por el director de la Revista Ciencia Digital.
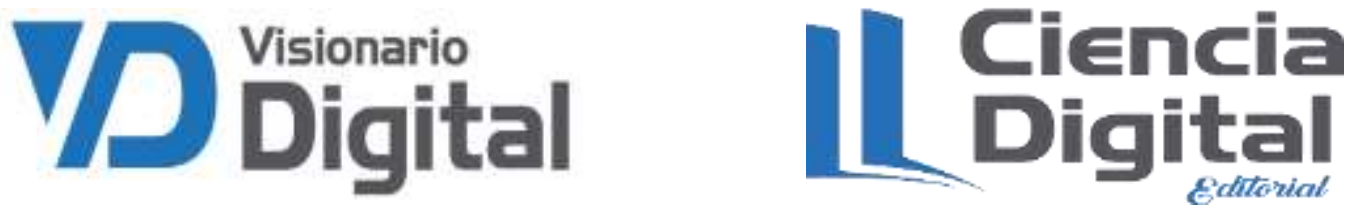